\title{
Frequency Locking of Josephson Junctions in a Surface Wave Resonator
}

\author{
Alexander M. Klushin, Edward Goldobin, Gennadiy A. Melkov, Oleksandr M. Ivanjuta, \\ Yury V. Eghorov, Kai Numssen, and Michael Siegel
}

\begin{abstract}
We report on the design, fabrication, and investigation of a series array of Josephson junctions embedded into the surface wave resonator. The resonator is fabricated by using $\mathrm{An}-\mathrm{YBa}_{2} \mathrm{Cu}_{3} \mathrm{O}_{7}$ bilayer on yttria-stabilized zirconia bicrystal substrate. The arrays were incorporated into the resonator by meandering the bilayer across a grain houndary. The circuit design and the excitation of the proper resonant mode provides series de biasing and parallel ac biasing of the JJs array. Experimental results show that microwave current is distributed uniformly along the whole $8 \mathrm{~mm}$ long array. In the vicinity of the resonant frequency, almost no difference between the microwave currents in the junctions was found.
\end{abstract}

Index Terms-Arrays, Josephson junctions, resonators, superconducting devices.

\section{INTRODUCTION}

$\mathrm{F}_{\mathrm{b}}^{\mathrm{se}}$ requency locking of the Josephson junctions (JJ) array by external microwave current is a principal requirement for the proper operation of metrological devices such as voltage standards, digital to analog converters, voltmeters, etc.[1]. Series arrays of high-temperature superconducting (HTS) bicrystal JJs, capable to operate at liquid nitrogen temperatures could be used in the new generation of such instruments [2], [3].

Recently, microwave properties of the surface wave resonators (SWR) were described [4]. An important feature of surface waves is the concentration of an electromagnetic field near the continuous (non patterned) superconducting film located on the dielectric substrate. The high surface current density and the same field distribution in the SWR and in the rectangular waveguide (fundamental mode $H_{10}$ ) makes the SWR, placed inside the waveguide, easy to excite and very attractive for $J J$ irradiation. The SWR was fabricated by using a $\mathrm{Au}-\mathrm{YBa}_{2} \mathrm{Cu}_{3} \mathrm{O}_{7-\delta}(\mathrm{Au}-\mathrm{YBCO})$ bilayer on an yttria-stabilized zirconia (YSZ) bicrystal substrate. The JJ

Manuscript received September 18,2000. This work was supported by the German BMBF under Grant No. 13N7534/1

A. M. Klushin, E. Goldobin, M. Siegel are with Forschungszentrum Jülich GmbH, Jülich, 52425 Germany (telephone: 49-2461-612347, e-mail: a.klushin( $($ z-juelich.de)

G. A. Melkov, O. M. Ivanyuta, Yu. V. Eghorov are with the Department of Radiophysics, Kiev Taras Shevchenko University, Kiev, 252033 Ukraine (telephone: +380-44-2660532, e-mail: melkov@boy.rpd.univ.kiev.ua).

K. Numssen is with the Physics Department E-10, Technical University of Munich, Garching, 85748 Germany (telephone: +49-89-1320912407, e-mail: kai.numssen@Physik.TU-Muenclien.de). array was embedded into the SWR by meandering a bilayer across the grain boundary (GB). For the excitation, GB was oriented co-axially with the waveguide. The substrate size perpendicular to the GB defines the resonant frequency of the SWR. The circuit design and the excitation of a proper SWR mode provides series dc biasing (by externally applied current) and parallel ac biasing (by the currents flowing in the SWR) of the JJs in the array.

In this paper we analyze the performance of the SWR with the strips and slits and compare it to that of an unpatterned SWR. General design criteria which allow to optimize the performance of the patterned SWR are presented. Further, we investigate experimentally the array of bicrystal JJs embedded into such a resonator and make conclusion about the applicability of such a system for metrological applications.

\section{Surface Wave Resonator Performance}

\section{A. Continuous film SWR}

The thin film SWR in the waveguide with dimensions $a \times b$ is shown in Fig.1. The resonant structure consists of dielectric substrate 1 with permittivity $\varepsilon$ and HTS film 2 with the length $l$ (along $y$ ) and the width $w$ (along $z$ ). The length and the width of the dielectric substrate could also be larger than $l$ and $w$ respectively. The distances between the resonator and the lower or upper broad waveguide walls were $\delta_{1}, \delta_{2}$, respectively. In the general case the angle $\varphi$ between the dielectric plate and the waveguide walls is not equal to $90^{\circ}$, as shown in Fig. 1. By changing this angle the coupling of SWR with the waveguide can be adjusted. The value of the coupling close to the critical one can be achieved at

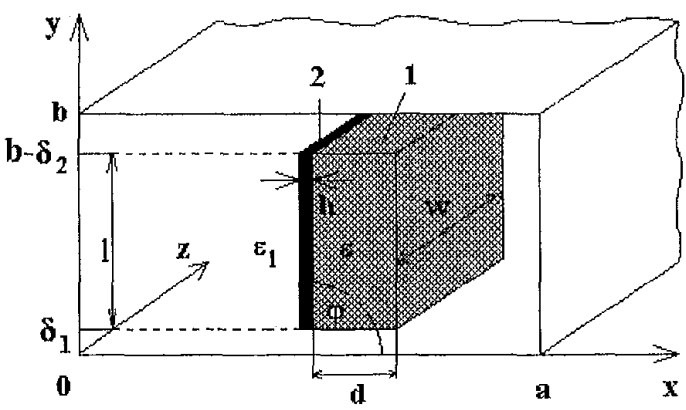

Fig. 1 Schematics of a surface wave resonator in a waveguide. 


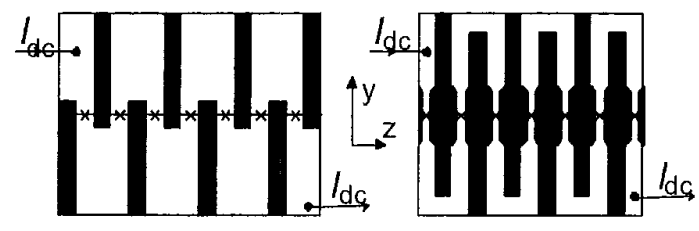

Fig. 2 Two typical topologies of the series connected JJs arrays. Black regions are the slits in HTS film, $-\mathrm{x}-$ are $\mathrm{JJS}$, and $\rightarrow$ indicates $I_{\mathrm{dtc}}$ bias $\mathrm{dc}$ current. Microwave bias current flows along slits in y direction.

$\varphi$ from $10^{\circ}$ to $20^{\circ}$ in the matched waveguide.

First, the unloaded quality factor $Q$ of the continuos film SWR was calculated and measured [4]. The typical values of $Q$ of about 1000-2000 were measured at the liquid nitrogen temperatures, when the SWR operates in the fundamental mode. Second, in the fundamental oscillation mode the microwave currents flow primarily in the metallic film along the $y$ direction. The current distribution along $z$-axis is quasi-uniform. This provides uniform ac biasing of JJs situated along GB in $z$ direction (Fig. 1).

To provide de series connection of bicrystal JJs the HTS film of the SWR is patterned as shown in Fig. 2. Many slits along $y$ directions and the non-uniformities (constrictions) as shown on Fig. 2 in the middle of the SWR can essentially decrease its performance: disturbing the uniform microwave current distribution, decreasing the SWR quality factor and shifting the resonant frequency. Below we discuss the influence of patterning on the SWR performance.

\section{$B$. SWR with slits in the conducting film}

We start with the analysis of the wave propagation in a flat dielectric waveguide covered with metallic strips as shown in Fig. 3. For such a structure the dispersion relation for the wave propagating parallel to the slits was found recently [5]. For a thin dielectric plate with $k d(\varepsilon-1)^{1 / 2}<1$, the effective permittivity can be approximated by the analytical expression

$\varepsilon_{1}(s)=1+k^{2} d^{2}\left(1-\frac{1}{\varepsilon}\right)^{2}-\frac{L \cdot(\varepsilon-1) \ln \left(\frac{1+\cos (\pi \cdot s)}{2}\right)}{4 \pi \cdot d\left(1+\frac{1}{\varepsilon}\right)+L \cdot \ln \left(\frac{1+\cos (\pi \cdot s)}{2}\right)}$

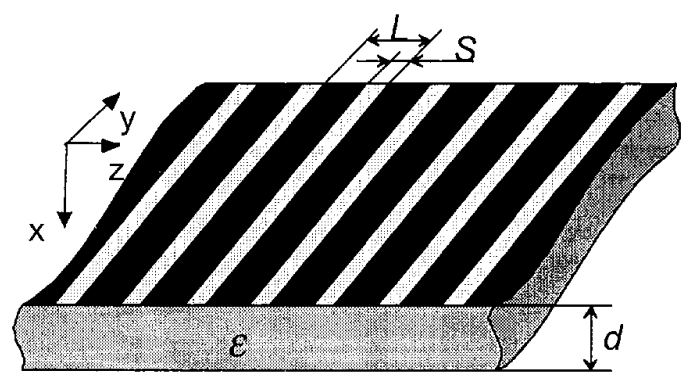

Fig. 3. A flat dielectric waveguide covered with metallic strips (black) separated with slits. The slits width is $S$, and the period of structure is $L$.
Here $k=2 \pi / \lambda, \lambda$ is the wavelength, $\varepsilon_{\|}=\varepsilon_{||}(s)$ is an effective permittivity, which depends on the normalized slit width $s=S / L$. For $s=0$, the last term of (1) vanishes [6]

$\varepsilon_{||}(0)=1+k^{2} d^{2}\left(1-\frac{1}{\varepsilon}\right)^{2}$

The relative change of $\varepsilon_{\|}$depending on $s$, $\eta_{\|}=\left[\varepsilon_{||}(s) / \varepsilon_{||}(0)\right]^{1 / 2}$, can be simply found for $S=L / 2$ from (1) and (2). For example, if $d=1 \mathrm{~mm}, k=22 \mathrm{~cm}^{-1}, \varepsilon=26$, $L=10 \mu \mathrm{m}$, then $\eta_{\|}(s=0.5)=0.993$. The above estimations show, that the effect of the longitudinal slits (Fig. 3) on $\varepsilon_{\mid !}$is very small and does not exceed $1 \%$ even if half of the conducting film is removed. This result suggests that the same behavior is also valid for the SWR shown in Fig. 2, since such an SWR is just a segment of the flat dielectric waveguide shown in Fig. 3. In particular, the following relation between resonant frequencies with slits $f_{\mathrm{r}}(\mathrm{s})$ and without slits $f_{\mathrm{r}}(0)$ can be proposed

$f_{\mathrm{r}}(s) / f_{\mathrm{r}}(0)=\left[\varepsilon_{\|}(0) / \varepsilon_{\|}(s)\right]^{1 / 2}$.

For the waves propagating in $z$-direction the influence of the slits is much larger. For a thin dielectric substrate the effective permittivity can be approximated by the following analytical expression

$\varepsilon_{\perp}(s)=1+k^{2} d^{2}\left(1-\frac{1}{\varepsilon}\right)^{2}+\frac{k^{2} d^{2}\left(1-\frac{1}{\varepsilon}\right)^{2}}{k^{2} d \frac{L}{4 \pi}\left(1-\frac{1}{\varepsilon}\right) \ln \left(\frac{1-\cos (\pi \cdot s)}{2}\right)-1}$

As expected, at $s=0 \quad \varepsilon_{\perp}=\varepsilon_{\|}$. The relative permittivity $\eta_{\perp}=\left[\varepsilon_{\perp}(s) / \varepsilon_{\perp}(0)\right]^{1 / 2}$, estimated for the parameters listed above, gives $\eta_{\perp}=0.75$ which corresponds to a larger frequency shift.

For the measurements we use two types of SWRs located in the middle of either X-band $\left(f_{\mathrm{r}} \sim 10 \mathrm{GHz}\right)$ or K-band $\left(f_{\mathrm{r}} \sim 30 \mathrm{GHz}\right)$ waveguides (Fig. 1). The first SWR consists of an alumina substrate $(e=9.8)$ with $l=10.3 \mathrm{~mm}, w=24 \mathrm{~mm}$, $d=0.5 \mathrm{~mm}$, covered by Al film with the thickness $h=10 \mu \mathrm{m}$. The second one consists of a YSZ substrate $(e=26)$, with $l=3 \mathrm{~mm}, w=10 \mathrm{~mm}, d=0.5 \mathrm{~mm}$, also covered with Al film of the thickness $h=10 \mu \mathrm{m}$. In each resonator a sequence of the slits (along $y$ direction) of the width $S \approx 100 \mu \mathrm{m}$ is made. The number of slits $N$ is equal to $1,3,7,15,31$ for the X-band and 1, 10, 32 for the K-band resonators. The normalized slit width $s$ grows with $N$.

The values of the normalized resonant frequency $f=f_{1}(s) / f_{r}(0)$ obtained experimentally for each value of $s$ are shown in Fig. 4. Solid squares correspond to the first type of the SWR. Solid crosses and diamonds correspond to the second type of SWR with orientation angle $\varphi \approx 15^{\circ}$ and $\varphi \approx 20^{\circ}$, respectively. It can be seen from Fig. 4 that $f$ almost does not depend on $s$ in accordance with (3). The measured values of $q=Q(s) / Q(0)$ are shown in Fig. 4. Solid circles 
correspond to the first type of the SWR. Solid up and down triangles correspond to the SWR of the second type with $\varphi \approx 15^{\circ}$ and $\varphi \approx 20^{\circ}$, respectively. Decreasing of $q$ with increasing of $s$ can be fitted in the whole investigated frequency band by the expression

$q=0.66+0.34 \cdot \exp (-s / 0.066)$

Since the snap formula (5) is the result of fitting of the experimental data obtained in a broad frequency band for different dielectrics and angles $\varphi$, we believe that this expression can be used for the quality factor estimation. In particular, it follows from (5) that even for larger $s$ the quality factor does not drop more than $40 \%$.

As it is mentioned above, the slits located transversally to the wave propagation direction considerably decrease the SWR performance. In the experiment, an X-band resonator with one slit along $z$ direction was used. The experimental dependencies of $f(s)$ and $q(s)$ are shown in Fig. 4 as open squares and circles, respectively. The resonant frequency shift achieves $10 \%$ already for $s \approx 0.05$. Moreover such a slit size drastically decrease the SWR quality. As can be seen from Fig. 4, a transversal slit with a width of order of $4 \cdot 10^{-3} l$ reduces $Q$ as much twice. The quality factor decrease can be fitted by the expression

$q=0.34 \cdot \exp (-s / 0.001)+0.66 \cdot \exp (-s / 0.027)$,

which is shown in Fig. 4 as a dashed line. The constrictions in superconducting strips (see Fig.2) in many respects are similar to the transversal slit. Therefore, such constrictions must be excluded from the topology of the SWR.

\section{Multimode Surface Wave Resonator}

With the increase of the dielectric thickness $d$ and resonator width $w$ multiple modes can be excited in the SWR, which decreases its quality. On the other hand a large width is essential to embed a large number of JJs into the SWR. Below we discuss the methods which allow to reduce the number of spurious modes.

To suppress $\mathrm{H}$ oscillations and the $\mathrm{E}$ oscillations with electric field variations along $x$-axes in the dielectric, the

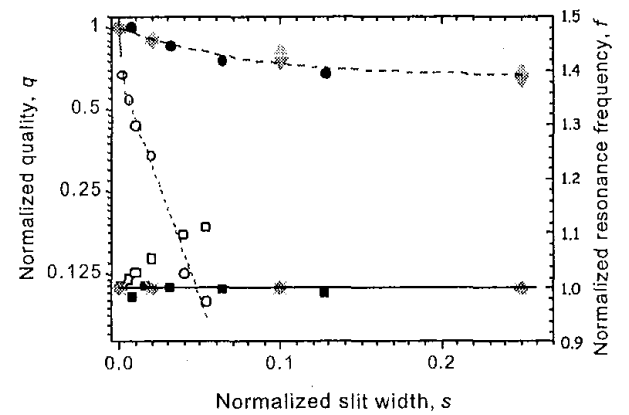

Fig. 4. The dependence of normalized resonant frequency $f=f(s) / f(0)$ and normalized quality factor $q=Q_{r}(s) / Q,(0)$ on normalized slit width $s$. substrate thickness $d$ should satisfy the known relation $k d(\varepsilon-l)^{1 / 2}<1$, i.e. YSZ substrates with $\varepsilon=26$ can be used in K-band, only if $d<250 \mu \mathrm{m}$.

At small $d$ there are only $\mathrm{E}$ modes with the different number of variations of the electric field, $E_{\mathrm{n} \mid m}$ and microwave current $j$ along $x, y, z$ axes. Note that for the vanishing $d$ and at the frequency corresponding to the resonant mode $n=l=1$. As an example, in Fig. 5 we show the measured dependence of the SWR resonant frequencies (for Al plate, $d=0$ ) on its width $w$. The fundamental mode $m=0$ has quasi-uniform current distribution along $z$-axis, $j_{z}=0$. The higher-order modes with $m \geq 1$ have a current component flowing in $z$ direction, $j_{z} \neq 0$. Therefore the topologies shown in Fig. 2 will effectively suppress highorder oscillations and naturally support the fundamental mode $\mathrm{E}_{110}$ on the background of spurious oscillations.

\section{JOSEPHSON JUNCTIONS ARRAYS IN SWR}

Josephson junctions arrays embedded in the SWR were fabricated on bicrystal YSZ substrates with misorientation angles $19^{\circ}$ or $24^{\circ}$. Epitaxial YBCO films were formed by reactive high-oxygen-pressure metal co-evaporation, by using a rotating substrate holder. The details of the intermittent film deposition scheme have been published previously [7]. In-situ evaporated, thin gold films were used as a shunt layer [2]. The substrate width along $z$-axis is $w=10 \mathrm{~mm}$. The substrate length along $y$ directions, $l=2.8 \mathrm{~mm}$, specifies the resonant frequency of the halfwave SWR in K-band. SWR is represented as a periodical strip system (Fig. 6) connected in series with $4 \mu \mathrm{m}$ wide bridges. The JJs were formed in the place of intersection of the bridge and GB (Fig. 6). The strips have the critical current, which is much larger than that of JJs, and are used to supply dc bias current and to measure the dc voltage across every $\mathrm{JJ}$ in the array. Arrays with 4 and $11 \mathrm{JJs}$ were fabricated. The periodic structure parameters were $L=1.68 \mathrm{~mm}, s \cong 0.009$ in the first case and $L=0.75 \mathrm{~mm}$, $s \cong 0.02$ in the second one. In both cases the distance between the first and the last junctions was about $8 \mathrm{~mm}$. Both samples were measured and have demonstrated approximately the same results. Therefore we are discussing

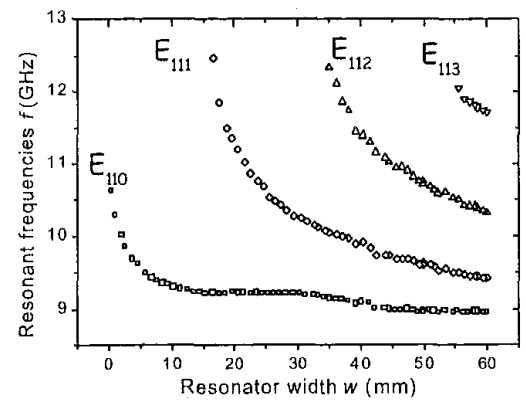

Fig. 5. Resonant frequencies of Al film $\left(l=12.2 \mathrm{~mm}, \varphi=12^{\circ}\right)$ SWR without dielectric, located in the center of the $\mathrm{K}$-band waveguide. 


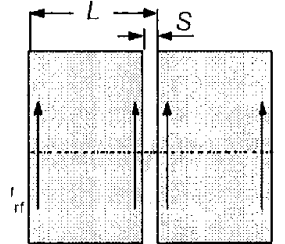

(a)

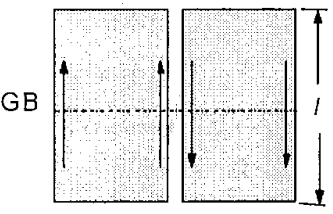

(b)
Fig. 6 Schematics of metallic strips connected in series with Josephson bridges. Arrows shows microwave current passing if neighboring strips resonate in-phase (a) or out-of-phase (b).

now only the data obtained for 4 junctions array. The measured SWR quality factor was $Q \cong 500$ without attached bias leads. The connection of the dc bias leads decreases $Q$ considerably.

The curve (a) in Fig. 7 shows the $I-V$ curve for 4 in series connected junctions without applied microwave power. The minimum and maximum critical currents among the JJs in the array were $I_{\text {cmin }}=560 \mu \mathrm{A}$ and $I_{\text {cmax }}=870 \mu \mathrm{A}$. The maximum value of the characteristic voltage was $V_{\mathrm{c}} \cong 80 \mu \mathrm{V}$ at $78 \mathrm{~K}$. To supply ac bias, the microwave signal with the frequency $f_{\mathrm{r}}=32950.8 \mathrm{MHz}$, which coincides with the resonant frequency of SWR, was applied. The amplitude of the first current step reached its maximum value $\Delta I_{\mathrm{c}}=I_{\mathrm{cmin}}$ at $P \approx 0.2 \mathrm{~mW}$. This means that the microwave current is distributed very uniformly along the whole $8 \mathrm{~mm}$ long array as it should be when the SWR operates in the fundamental mode.

Nevertheless it is interesting how slits and bridges affect the ac current distribution in our structure. Fitting the experimental $I-V$ curves by using equations of RSJ model, the amplitude of ac bias current was estimated as $I_{\mathrm{ac}} \approx 1.5 \mathrm{~mA}$. On the other hand, the total ac current in the SWR at the level of absorbed power of $P=1 \mathrm{~mW}$ is equal to $0.08 \mathrm{~A} / \mathrm{mm}$ for the strip width $w \approx 1.7 \mathrm{~mm}$ [4]. Comparing the value of the microwave current in the strip with the value of $l_{\text {ac }}$ calculated above, we find that approximately $20 \%$ of the ac current in the resonator passes through the junctions.

In general, the detailed picture of the current distribution in the system shown in Fig. 6 is not well understood. In particular the question whether the neighboring strips resonate in-phase [Fig. 6 (a)] or out-of-phase [Fig. 6 (b)] is not answered. If the currents were flowing out-of-phase in

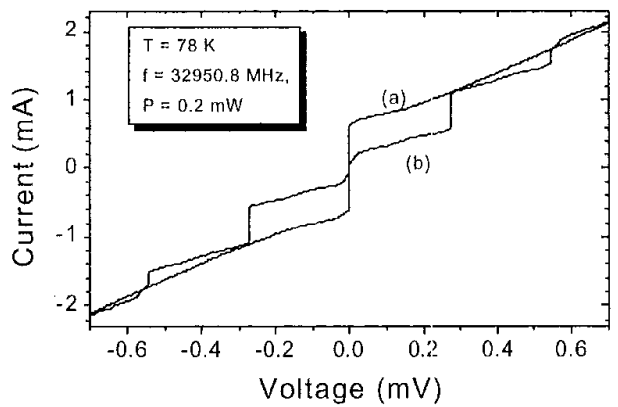

Fig. 7 I-V Curve of 4 series connected JJs (a) without microwave power and (b) with microwave power. the neighboring strips, they would short via the bridge containing $\mathrm{JJ}$. In this case the junction would be biased by the ac current with a spatial profile which is the odd function of $z$. Here $z$ is the coordinate along the junction and $z=0$ is in the center of it. The numerical simulation shows that junction with such an ac bias current profile exhibits the even current steps on the $I-V$ curve, which have much larger amplitude than the corresponding odd steps. On the other hand, in the experiment this kind of behavior was not observed. If the neighboring strips oscillate in-phase, the ac current should not flow through the junction at all, which is again in contradiction with out data.

\section{CONCLUSION}

We have shown the frequency locking of JJs embedded in the SWR. Different topologies providing de series connections of JJS are discussed. In all the cases the SWR were patterned so that the resulting structure consists a number of parallel strips separated by slits. General design criteria were found for the resonant structure parameter optimization. It was shown theoretically and confirmed experimentally that for the longitudinal slits the resonant frequency does not depend on $s$, and the quality factor decreases slightly with the increasing $s$. On the contrary, the slits located transversally to the direction of the wave propagation decrease the SWR performance considerably. The SWR quality decreases especially strongly. The multimoding in the SWR was considered and the conditions of suppressing the spurious modes were found. It was shown that the discussed topologies naturally support the fundamental mode $\mathrm{E}_{110}$ and reject the others. The Josephson junction array embedded into the SWR has been investigated. Experimental data present evidence that the microwave current distribution in the SWR is uniform and corresponds to the fundamental mode. These results make JJ arrays embedded into the SWR a promising system for applications in the quantum voltage metrology.

\section{References}

[1] J. Niemeyer, "Josephson arrays for de and ac metrology", Supercond Sci. Technol., vol. 13, pp. 546-560, 2000.

[2] A. M. Klushin, W. Prusseit, E. Sodtke, S. I. Borovitskii, L. E. Anatuni, and H. Kohistedt, "Shunted bicrystal Josephson junctions arrays for voltage standards", Appl. Phys. Lett., vol. 69, pp. 1634-1636, July 1996.

[3] A. M. Klushin, C. Weber, H. Kohlstedt, R. K. Starodubrovskii, A. Lauer, I. Wolff, and R. Semerad, "New microwave circuits for programmable voltage standards using high-temperature Josephson junction arrays", IEEE Trans. Appl. Supercond, vol. 9, pp.4158-4161, June 1999.

[4] G. A. Melkov, Y. V. Eghorov, O. M. Ivanjuta, V. Y. Malishev, H. K. Zeng, K. H. Wu, and J. Y. Juang, "HTS surface wave resonators", J. Supercond., vol. 13, pp. 95-100, Jan. 2000.

[5] G. A. Melkov, Y. V. Eghorov, O. M. Ivanjuta, A. M. Klushin, M. Siegel, and R. Semerad, "Josephson generation of junctions array in surface wave resonator", submitted for publication.

[6] G. A. Melkov, and Y. V. Eghorov, "Swihart waves and surface plasmons in a plain-parallel superconducting transmission line", Low Temp. Phys., vol. 26, pp. 108-114, Febr. 2000.

[7] H. Kinder, P. Berberich, W. Prusseit, S. Rieder-Zecha, R. Semerad, and B. Utz, "YBCO film deposition on very large areas up to $20 \times 20 \mathrm{~cm}^{2}$ " Physica C, vol C 282-287, pp, 107-110, 1997. 